This document is the Accepted Manuscript version of a Published Work that appeared in final form in Journal of Physical Chemistry Letters, copyright (c) American Chemical society after peer review and technical editing by the publisher. To access the final edited and published work see

https://pubs.acs.org/doi/abs/10.1021/acs.jpclett.7b01024

\title{
Non-zero Ideal Gas Contribution to the Surface Tension of Water
}

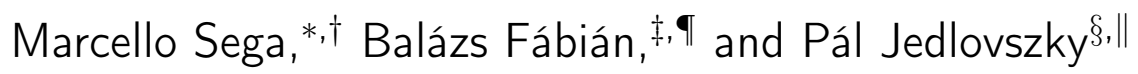 \\ †University of Vienna, Boltzmangasse 5, A-1090 Vienna, Austria \\ †Institut UTINAM (CNRSUMR6213), Université Bourgogne Franche Comtè 16 route de \\ Gray, F-25030 Besançon, France \\ 【Department of Inorganic and Analytical Chemistry, Budapest University of Technology \\ and Economics, Szt. Gellért tér 4, H-1111 Budapest, Hungary \\ $\S$ Department of Chemistry, Eszterházy Károly University, Leányka u. 6, H-3300 Eger, \\ Hungary \\ ||MTA-BME Research Group of Technical Analytical Chemistry, Szt. Gellért tér 4, H-1111 \\ Budapest, Hungary \\ E-mail: marcello.sega@univie.ac.at
}




\begin{abstract}
Surface tension, the tendency of fluid interfaces to behave elastically and minimize their surface, is routinely calculated as the difference between the lateral and normal components of the pressure or, invoking isotropy in momentum space, of the virial tensor. Here we show that the anisotropy of the kinetic energy tensor close to a liquid/vapour interface can be responsible for a large part of its surface tension (about 15\% for water, independently from temperature).
\end{abstract}

\title{
Graphical TOC Entry
}

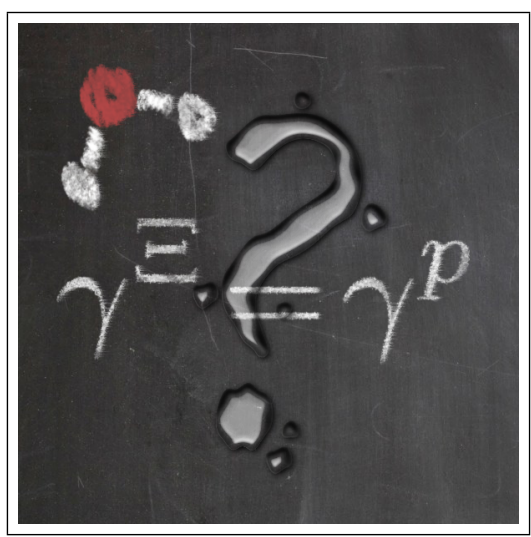


The surface tension of a fluid can be obtained in several ways from the microscopic variables describing the system: the so-called mechanical route links the surface tension of a planar interface to the imbalance between the normal $\left(p_{N}\right)$ and lateral $\left(p_{T}\right)$ components of the pressure tensor, $\gamma^{p}=\int_{-\infty}^{\infty} p_{N}-p_{T}(z) d z$. In a periodic system of size $L$ one can use the volume average of the pressure tensor to write the surface tension as $\gamma^{p}=L\left(p_{N}-p_{T}\right) / 2$, where the factor $1 / 2$ takes into account the presence of two interfaces. For a system of point-like particles, the pressure tensor $\mathbf{p}$ can be accessed through the virial route, ${ }^{1,2} \mathbf{p}=$ $2(\mathbf{K}-\boldsymbol{\Xi}) / V$, where $V$ is the system volume, $\mathbf{K}=1 / 2 \sum_{i} m_{i} \mathbf{v}_{i} \otimes \mathbf{v}_{i}$ is the kinetic energy tensor (corresponding to the ideal gas contribution) and $\boldsymbol{\Xi}$ is the virial tensor, which, for pairwise-additive forces $\mathbf{f}_{i j}$ between particle $i$ and $j$ can be written as $\boldsymbol{\Xi}=-1 / 2 \sum_{i>j} \mathbf{r}_{i j} \otimes$ $\mathbf{f}_{i j}$. If no constraints are present in the system, it is possible to invoke the equipartition theorem, ${ }^{3}\langle x \partial H / \partial x\rangle=k_{B} T$ ( $H$ being the Hamiltonian, $k_{B}$ Boltzmann's constant, and $T$ the absolute temperature) for the elements of the kinetic energy tensor, and write the (average) pressure tensor as $\mathbf{p}=\rho k_{B} T \mathbf{1}-2 \boldsymbol{\Xi} / V$, where $\rho$ is the number density of atoms, and $\mathbf{1}$ is the unit tensor. This allows to write an alternative expression for the surface tension, $\gamma^{\Xi}=-L /(2 V)\left(\Xi_{N}-\Xi_{T}\right)$, which is, on average, completely equivalent to the one involving the full pressure tensor, $\gamma^{p}$, but has the advantage of not requiring to sample velocities. The equivalence $\gamma^{p}=\gamma^{\Xi}$, in other words, means that only the virial part of the pressure contributes to the surface tension, whereas the ideal gas contribution is zero. This, one should stress, is only true in absence of constraints.

In water, however, the softest internal degree of freedom, the bending mode, has a frequency of about $1640 \mathrm{~cm}^{-1}$. This corresponds, at room temperature, to an activation energy for the first excited state of roughly $7.8 k_{B} T$, and a corresponding average energy of about $3 \times 10^{-3} k_{B} T$. Excited stretching modes have even higher energies, and in this sense water molecules are, for the sake of computing the surface tension, just rigid bodies. In this case, the partition function is not separable any more into a kinetic and a configurational part, ${ }^{4-7}$ and the corresponding constraints acting on Cartesian coordinates and velocities appear in 


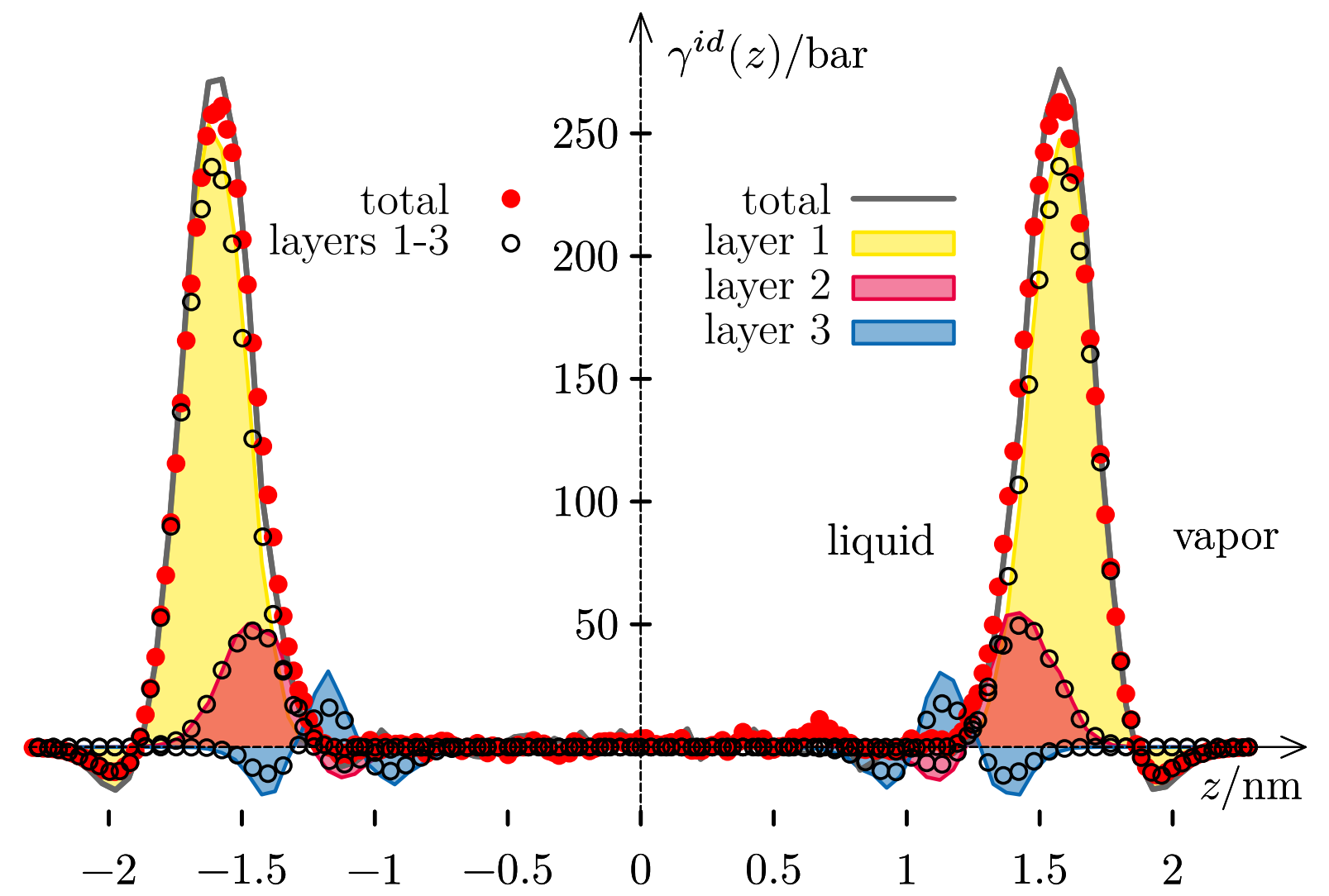

Figure 1: Ideal gas rotational contribution $\gamma^{\text {id }}$ to the surface tension profile as computed from the kinetic energy tensor (solid line) and from Eq. (1) (full circles); contribution of successive molecular layers calculated from the kinetic energy tensor (shaded areas) and from Eq. (1) (open circles).

the expression for the pressure tensor, as it is well known for liquid crystals. ${ }^{8}$ Small molecular liquids, on the contrary, do not usually enjoy long-range order in the bulk, and the effect of this coupling vanishes due to isotropy. The presence of an interface, introducing a preferential direction in the system, can, however, change this picture substantially, so that in principle the equivalence between $\gamma^{p}$ and $\gamma^{\Xi}$ is not guaranteed any more, and a finite ideal gas contribution to the surface tension could appear.

In the course of extensive testing for the calculation of the surface tension of the SPC/E water model ${ }^{9}$ we found that the difference between $\gamma^{p}$ and $\gamma^{\Xi}$ amounts to about $15 \%$ at ambient temperature. We were able to reproduce the same discrepancy with different software packages, integrators (including quaternions to describe the rigid body motion), thermostats, 
and electrostatic treatments. In particular, we reproduced the same behaviour also in the microcanonical ensemble, guaranteeing conservation of the total energy to within at least $1 \mathrm{ppm}$, with no evident drift within one nanosecond of simulation. The asymmetry of the kinetic energy tensor does not show any dependence on system size or timestep, ruling out other known effects that seemingly violate equipartition. ${ }^{10-14}$ Although this effect does not depend on the implementation of the constraints, substituting them with harmonic springs completely removes the asymmetry, and allows to recover the equality $\gamma^{p}=\gamma^{\Xi}$, confirming that it is the rigid arrangement of atoms in the molecules that is at the origin of this apparent violation of equipartition.

Since the properties of water molecules at the liquid/vapour interface differ from the bulk ones only in the first two or three layers, ${ }^{15,16}$ it is reasonable to expect the kinetic energy tensor to be anisotropic only in proximity to the interface. The kinetic energy tensor is a well-defined local quantity, therefore it is possible to calculate, without the ambiguity that characterizes the configurational part of the pressure, ${ }^{17}$ its profile along the surface normal, $\mathbf{K}(z)=\left\langle 1 / 2 \sum_{i} m_{i} \mathbf{v}_{i} \otimes \mathbf{v}_{i} \delta\left(z-z_{i}\right)\right\rangle$, where it is assumed that the center of mass of the liquid phase is shifted at the origin of the coordinate system. The difference between the normal and the lateral components of $\mathbf{K}(z)$ can be used to compute the ideal gas contribution $\gamma^{\text {id }}(z)=\gamma^{p}(z)-\gamma^{\Xi}(z)$ to the surface tension, shown in Fig. 1, which is indeed concentrated in proximity to the interfaces. The ideal gas contribution originates only from the rotational degrees of freedom of the molecules, as the translational ones (that is, the molecular centers of mass positions) behave isotropically.

The coupling of the kinetic degrees of freedom to the positional ones can be exploited to derive an expression for the ideal gas surface tension profile of rigid molecules, as a function of molecular orientations. Using the atomic positions $\mathbf{r}_{i}^{\prime}$ and the angular velocity vector $\omega$ in the molecular co-moving frame, the velocity of each atom in the lab frame can be written as $\mathbf{v}_{i}=\omega \times \mathbf{R}(\phi, \theta, \psi) \mathbf{r}_{i}^{\prime}$, where $\mathbf{R}$ is the Euler rotation matrix parametrized by the three Euler angles $\phi, \theta, \psi$. With the help of the equipartition theorem, quadratic 


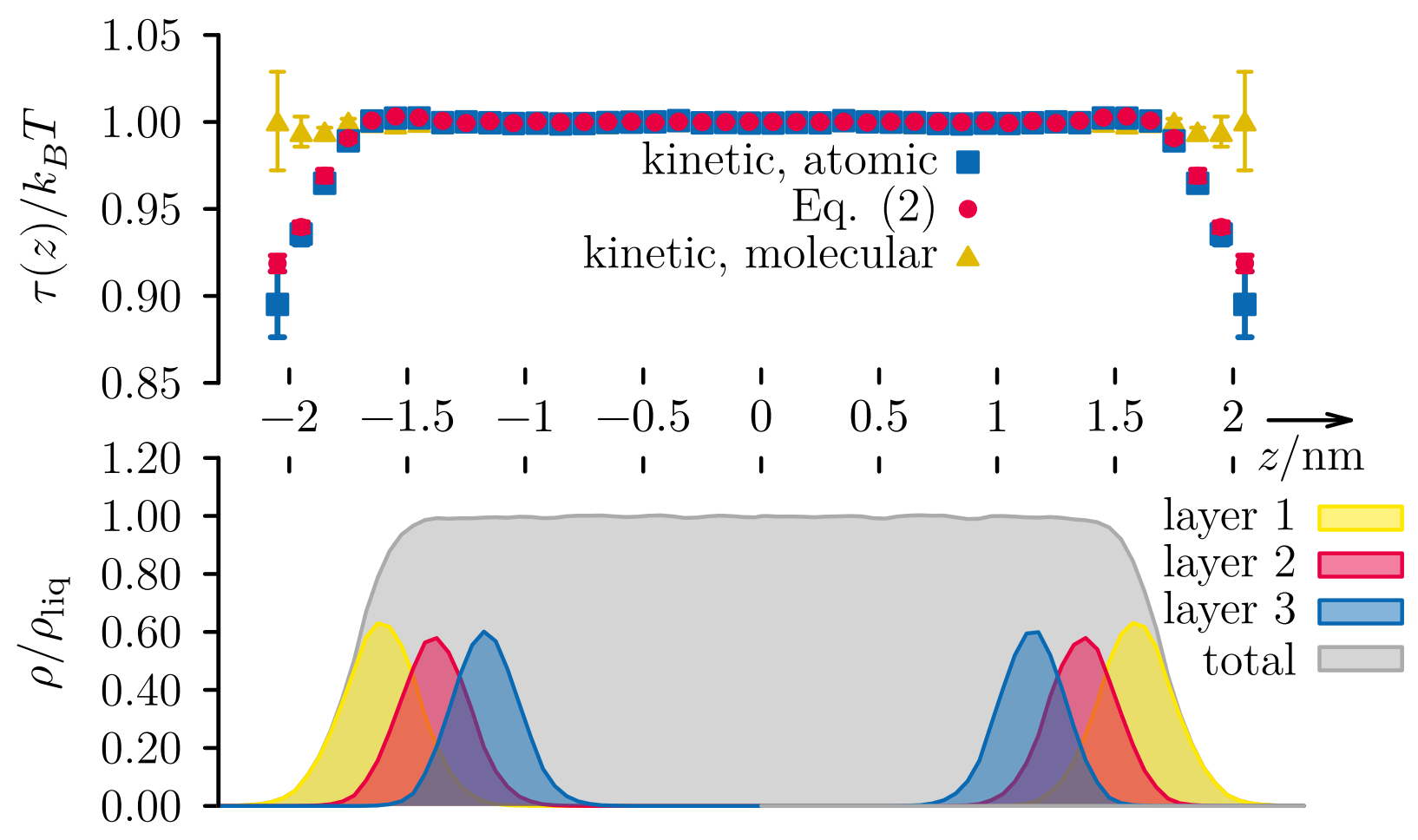

Figure 2: Upper panel: kinetic energy density profile $\tau(z)$ calculated using the atomic expression $k_{B} \tau(z)=\operatorname{Tr} \mathbf{K}(z) / \rho(z)$ (blue squares), using Eq.2 (red circles), and using the molecular expression (yellow triangles), as described in the text. Lower panel: atomic density profile of the whole system and of the first three layers, normalized to the density in the liquid region.

terms in the components of $\omega$ appearing in the average can be expressed as functions of the components of the inertia tensor $\mathbf{I}$ associated to the molecular structure. For a symmetric top, corresponding to the case of a linear molecule, such as $\mathrm{O}_{2}$, where $\mathbf{I}=\operatorname{diag}(I, I, 0)$, the ideal gas surface tension contribution of the $i$-th atom is $\gamma_{i}^{\text {id }}=-k_{B} T L P_{2}\left(\cos \theta_{i}\right) / 2 \mathrm{~V}$, where $P_{2}(\cos \theta)=3 / 2 \cos ^{2}(\theta)-1 / 2$ is the second order Legendre polynomial and $\theta_{i}$ identifies the angle between the molecular axis and the macroscopic surface normal $\hat{z}$. For a flat, asymmetric top like water, initially laying in the $x z$ plane with the dipole vector oriented along the $z$ axis, $\mathbf{I}=\operatorname{diag}\left(I_{x}, I_{x}+I_{z}, I_{z}\right)$, and the ideal gas contribution of the $i$-th atom located, in the molecular frame, at $\left(x_{i}^{\prime}, 0, z_{i}^{\prime}\right)$, takes the form

$$
\gamma_{i}^{\mathrm{id}}=\frac{L k_{B} T m_{i}}{2 V I_{x} I_{y} I_{z}}\left[f(\mathbf{I}) P_{2}(\cos \theta)+g(\mathbf{I}) P_{2}(\cos \delta)\right],
$$




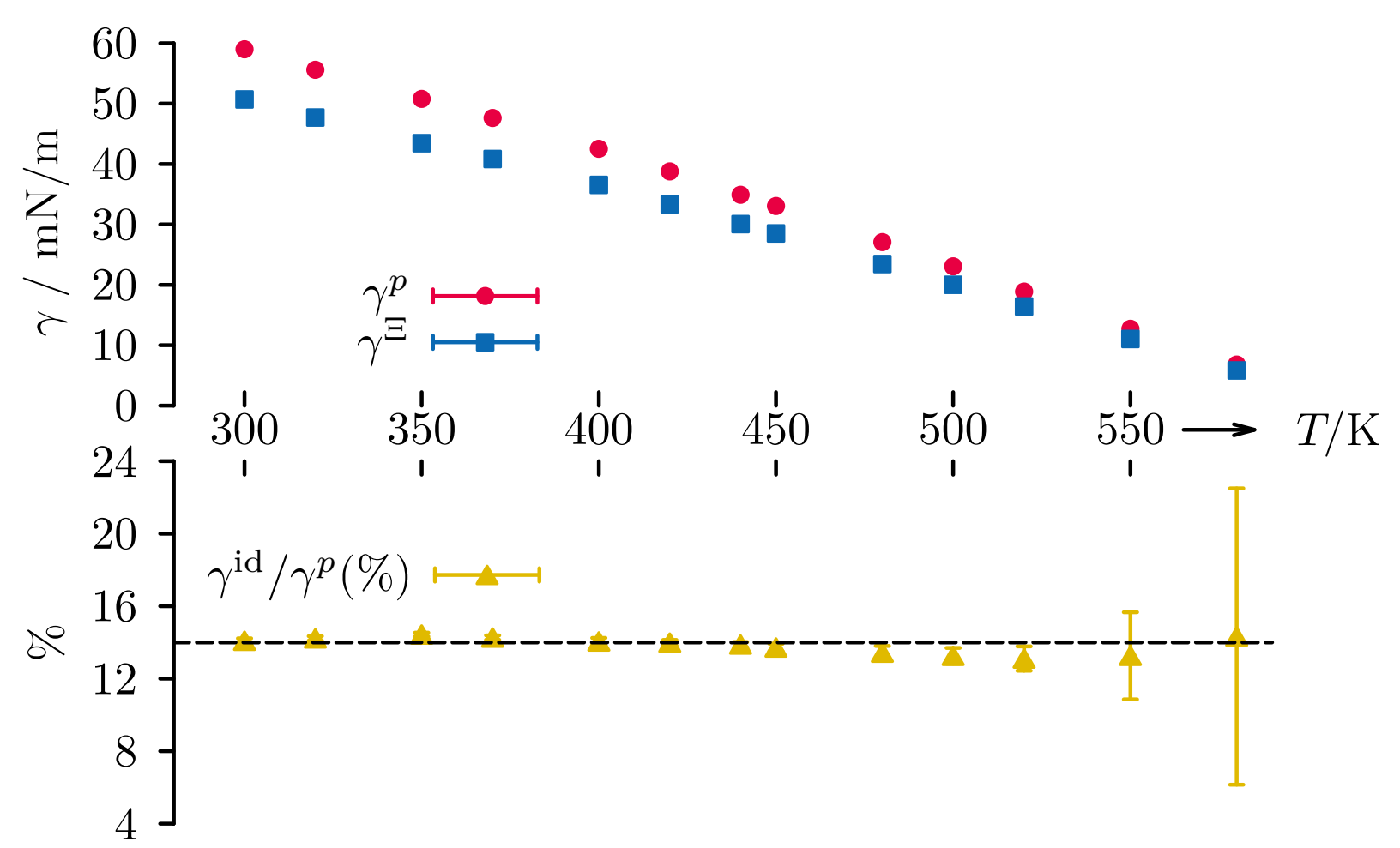

Figure 3: Upper panel: surface tension $\gamma^{p}$ and virial contribution $\gamma^{\Xi}$ as a function of temperature. Lower panel: ratio $\gamma^{\text {id }} / \gamma^{p}=1-\gamma^{\Xi} / \gamma^{p}$. Error bars are always smaller than the symbols in the upper panel, and are for all temperatures of the order of $0.1-0.2 \mathrm{mN} / \mathrm{m}$.

Table 1: Temperature-independent ratio $\gamma^{\text {id }} / \gamma^{p}(\%)$ for different water models.

\begin{tabular}{ccccccc}
\hline \hline SPC & SPC /E & TIP3P & TIP4P & TIP4P-2005 & TIP5P & TIP5P/E \\
$14.3(2)$ & $15.0(1)$ & $15.4(2)$ & $11.5(1)$ & $11.4(1)$ & $9.2(5)$ & $8.7(4)$ \\
\hline
\end{tabular}

where $f(\mathbf{I})=I_{x} I_{y}\left(x_{i}^{\prime 2}-z_{i}^{\prime 2}\right), g(\mathbf{I})=I_{x} I_{y} x_{i}^{\prime 2}+I_{z}^{2} z_{i}^{\prime 2}$, and $\delta$, the angle between the molecular plane and the surface normal, is related to the Euler angles through the expression $\cos (\delta)=$ $\cos (\psi) \sin (\theta)$. The derivation of 1 can be found in the Supporting Information.

The surface tension profile calculated using Eq. (1) as $\gamma(z)=\sum_{i}\left\langle\gamma_{i} \delta\left(z-z_{i}\right)\right\rangle$ reproduces strikingly well the ideal gas rotational contribution obtained using the kinetic energy tensor, as reported in Fig. 1 with full circles and solid line, respectively. The integral of the two curves differ only by $0.5 \%$.

This shows that, in fact, equipartition is not violated, as it has been used to derive Eq. (1), taking into account the correlations between kinetic and positional degrees of freedom, introduced by the presence of constraints. The explicit relation between the ideal gas contribution 
and the molecular ordering can be now used to look from a different perspective some other results related to the surface tension. More precise structural information can be gained by identifying the molecules composing successive molecular layers below the interface, ${ }^{18,19}$ and calculating their contribution to the ideal surface tension $\gamma^{\text {id }}$. Such an decomposition, also shown in Fig.1, shows that the difference between the normal and lateral kinetic components of the stress tensor is indeed located mainly at the first molecular layer, with some minor deviations showing up in the second one, and with the third layer being already characterized by nearly zero-sum oscillations, which have a modest contribution to the total value of $\gamma^{\text {id }}$. We already observed this kind of oscillations in the full surface tension profile of water, ${ }^{16}$ but their physical significance was not clear because of the virial contribution to the pressure profile not being a well-defined quantity. ${ }^{20}$ The kinetic part of the profile, however, does not suffer from these interpretation problems, and the profile obtained by using Eq. (1) to compute the contribution of different layers (Fig.1, open circles) shows that the oscillations are the result of the correlation between molecular orientation and deviation from the mean layer position.

Not only the surface tension, but also the kinetic energy density shows departure from the constant value one would expect. For water molecules, the relation between kinetic energy $E$, number of atoms $N$ and temperature is $k_{B} T=\langle E / N\rangle$, which can be written naively in a local form as $k_{B} \tau(z)=\operatorname{Tr} \mathbf{K}(z) / \rho(z)$, with $\tau(z)$ representing the kinetic energy density profile. The profile so defined departs from the constant value $T$ in the proximity of the interface (see Fig. 2). This, again, is not a violation of the equipartition theorem, which can be used to derive the correct expression for the average kinetic energy contribution of the $i$-th atom in the water molecule

$$
e_{i}=m_{i} \frac{k_{B} T}{2}\left\{\frac{3}{M}+\frac{x_{i}^{\prime 2}}{I_{y}}+\frac{x_{i}^{\prime 2}}{I_{z}}+\frac{z_{i}^{\prime 2}}{I_{x}}+\frac{z_{i}^{\prime 2}}{I_{y}}\right\}
$$

where $M$ is the mass of the molecule, and the term $3 / M$ is the molecular center of mass 
velocity (translational) contribution to the atom's kinetic energy, while the remaining terms are the rotational contributions (interestingly, for the symmetric top, the rotational contribution of each atom is simply $k_{B} T / 2$ ). The atomic kinetic energy density profile can thus be written using only atomic positions as $k_{B} \tau(z)=\sum_{i}\left\langle e_{i} \delta\left(z-z_{i}\right)\right\rangle / \rho(z)$, and is shown in Fig. 2 to reproduce very well the kinetic definition. Obviously, this quantity does not correspond to the usual thermodynamic temperature, which is expected to be constant across the interface. The temperature so defined loses its meaning once correlations between momenta and atomic positions occur. However, by using a molecular-based definition of the kinetic energy, in which the translational and rotational contributions are concentrated at the centre of mass of the molecule, one obtains the expected constant profile, as shown also in Fig. 2.

As it is clear that the kinetic part of the pressure tensor is needed to compute the correct value of the surface tension if rigid molecules are present in the system, one might wonder if this can create problems for methods like Monte Carlo, which do not provide explicit access to momenta. In fact, if one wants to compute the surface tension through explicit calculation of the pressure tensor elements, there is no other way but to include the kinetic contribution through formulae like Eq. 1. However, this is not the only possible route to the surface tension: the test-area method ${ }^{21}$ (of which also local variants exist ${ }^{22}$ ), for example, follows a thermodynamic route to compute the surface tension as the limit towards vanishing crosssectional surface area perturbations $\Delta A$ (at constant volume) of the associated changes in Helmholtz free energy $F$, so that $\gamma=\lim _{\Delta A \rightarrow 0} \Delta F / \Delta A=-k_{B} T \ln \left\langle\exp \left(-\Delta U / k_{B} T\right)\right\rangle$, where $\Delta U$ is the change in potential energy between the perturbed and not perturbed state, and the ensemble average is performed over configurations sampled from the unperturbed state. Since this is (in the limit of small perturbations) the thermodynamic definition of the surface tension, one might expect it to yield the total surface tension, including the kinetic contributions. To test this, we computed the surface tension for water at $T=300 \mathrm{~K}$ using the test area method with area changes of $0.1,0.05$ and $0.01 \%$, respectively, and extrapolated the results to vanishing area changes using a linear fit, resulting in a surface tension estimate 
of $59.5 \pm 0.1 \mathrm{mN} / \mathrm{m}$. This has to be compared with the mechanical route results of $59.9 \pm$ $0.2 \mathrm{mN} / \mathrm{m}$ (full pressure tensor) and $51.2 \pm 0.2 \mathrm{mN} / \mathrm{m}$ (virial contribution only). The test area method is therefore an appropriate way to obtain the surface tension with Monte Carlo methods when rigid molecules are present.

Finally, we report a surprising result obtained from the analysis of the temperature dependence of the ideal gas contribution on the surface tension. Since the orientational preference of water molecules at the surface has to vanish when approaching the critical point, one can expect $\gamma^{\text {id }}$ to decrease when the temperature increases. The values of $\gamma^{p}$ and $\gamma^{\Xi}$ show, in fact, a similar decreasing pattern, reaching convergence as the temperature approaches the critical value, as shown in Fig. 3. What is quite remarkable, however, is that the relative contribution $\gamma^{\text {id }} / \gamma^{p}$ is, to a good approximation, independent of the temperature, and oscillates within few fractions of a percent around 15\%. We tested different rigid water models (SPC ${ }^{23} \mathrm{TIP} 3 \mathrm{P},{ }^{24} \mathrm{TIP} 4 \mathrm{P},{ }^{24} \mathrm{TIP} 4 \mathrm{P}-2005,{ }^{25} \mathrm{TIP} 5 \mathrm{P},{ }^{26} \mathrm{TIP} 5 \mathrm{P} / \mathrm{E}^{27}$ ), obtaining in all cases a temperature independent ratio of $\gamma^{\text {id }} / \gamma^{p}$, although the value itself is model-dependent, ranging from about 9 to $15 \%$, as it is shown in Tab. 1. There is, therefore, a direct proportionality between the orientational order of molecules, of which $\gamma^{\text {id }}$ is representative, and the surface tension of the system, $\gamma^{p} \propto \gamma^{\text {id }}$.

\section{Methods}

Simulations have been performed using the GROMACS 5.1, ${ }^{28}$ LAMMPS, ${ }^{29}$ and ESPResSo ${ }^{30}$ molecular simulation packages using either single or double precision. Water molecules have been kept rigid by either solving the constrained equation of motion using the SHAKE ${ }^{31}$ or SETTLE $^{32}$ algorithms or, in case of ESPResSo, by solving the rigid-body dynamics using quaternions. The difference between $\gamma^{\Xi}$ and $\gamma^{p}$ has been shown to persist independently from short-range forces at the cutoff distance (force truncation vs. potential shift); mesh size and accuracy in the smooth Particle Mesh Ewald ${ }^{33}$ (sPME) or Particle-Particle Particle-Mesh 
Ewald ${ }^{34}$ method; number of reciprocal vectors and $\beta$ parameter in plain Ewald method); integration timestep from 0.1 fs to $1 \mathrm{fs}$; simulation box size; type of thermostat (Berend$\operatorname{sen}^{35}$ vs. Nosé-Hoover ${ }^{36,37}$ ) ; ensemble (microcanonical vs. canonical). The actual value of the surface tension depends on the short-range interactions cut-off value as well as on the parameters used to compute the electrostatic interaction. The data reported in Figs. 1 and 2 are obtained by simulating 1488 water molecules in a $3.6 \times 3.6 \times 9.6 \mathrm{~nm}^{3}$ simulation box with the velocity-Verlet algorithm, smoothly switching the short-range forces to zero in the interval between 1.55 and $1.6 \mathrm{~nm}$, using sPME with an accuracy of $10^{-8}$ for the real part of the screened electrostatic potential at $1.55 \mathrm{~nm}$ and a reciprocal space grid of $128 \times 128 \times 256$, the Nosé-Hoover thermostat with the reference temperature of $T=300 \mathrm{~K}$ and relaxation constant $0.5 \mathrm{ps}$, and an integration timestep of $0.1 \mathrm{ps}$. The data reported in Fig. 3 and Table 1 were obtained by simulating 1000 water molecules in a $3 \times 3 \times 3.5 \mathrm{~nm}^{3}$ simulation box with the leapfrog algorithm, the sPME algorithm with an accuracy of $10^{-5}$ at the real- space cut-off of $1.3 \mathrm{~nm}$, which is also used as a cut-off for the van-der-Waals interactions, without using switching functions for the force, and an integration step of $1 \mathrm{fs}$. The calculation of the surface tension profiles and the layer-by-layer analysis have been performed using the ITIM algorithm ${ }^{18,38}$ for the identification of surface molecules, with a probe sphere radius of $0.2 \mathrm{~nm}$. The liquid and gas phases have been distinguished before determining the layer structure using a cut-off $(0.35 \mathrm{~nm})$ based cluster search that associates the liquid phase to the largest cluster in the system. ${ }^{39}$ The low inaccuracies for the values of the surface tension as well as smooth profiles were obtained by analysing 30000 samples over $3 \mathrm{~ns}$ for the data reported in Figs. 1 and 2, and 50000 samples over 50 ns for each system and temperature for the data reported in Fig. 3 and Tab. 1. 


\section{Acknowledgements}

The authors acknowledge useful discussions with Giovanni Ciccotti, Simone Melchionna and Francesco Sciortino. This work has been supported by the Marie-Skłodowska-Curie European Training Network COLLDENSE (H2020-MSCA-ITN-2014 Grant No. 642774), the Hungarian NKFIH foundation (Project No. 119732) and the Action Austria-Hungary Foundation (Project No. 93öu3).

Supporting Information. Derivation of the equations for the ideal gas surface tension contribution.

\section{References}

(1) Hansen, J. P.; McDonald, I. R. Theory of simple liquids, 2nd ed.; Academic Press: London, UK.

(2) Hünenberger, P. H. Calculation of the group-based pressure in molecular simulations. I. A general formulation including Ewald and particle-particle-particle-mesh electrostatics. J. Chem. Phys. 2002, 116, 6880-6897.

(3) Tolman, R. C. A general theory of energy partition with applications to quantum theory. Phys. Rev. 1918, 11, 261-272.

(4) Ryckaert, J. P.; Ciccotti, G. Introduction of Andersen's demon in the molecular dynamics of systems with constraints. J. Chem. Phys. 1983, 78, 7368.

(5) Ciccotti, G.; Ryckaert, J. P. Molecular dynamics simulation of rigid molecules. Comput. Phys. Rep. 1986, 4, 346-392.

(6) Sprik, M.; Ciccotti, G. Free energy from constrained molecular dynamics. J. Chem. Phys. 1998, 109, 7737-7744. 
(7) Melchionna, S. Constrained systems and statistical distribution. Phys. Rev. E 2000, $61,6165-6170$.

(8) Chaikin, P. M.; Lubensky, T. C. Principles of condensed matter physics; Cambridge Univ Press: Cambridge, 1995.

(9) Berendsen, H. J. C.; Grigera, J. R.; Straatsma, T. P. The missing term in effective pair potentials. J. Phys. Chem. 1987, 91, 6269-6271.

(10) Shirts, R. B.; Burt, S. R.; Johnson, A. M. Periodic boundary condition induced breakdown of the equipartition principle and other kinetic effects of finite sample size in classical hard-sphere molecular dynamics simulation. J. Chem. Phys. 2006, 125, 164102.

(11) Corti, D. S.; Uline, M. J.; Siderius, D. W. On the generalized equipartition theorem in molecular dynamics ensembles and the microcanonical thermodynamics of small systems. J. Chem. Phys. 2008, 128, 124301.

(12) Eastwood, M. P.; Stafford, K. A.; Lippert, R. A.; Jensen, M. Ø.; Maragakis, P.; Predescu, C.; Dror, R. O.; Shaw, D. E. Equipartition and the calculation of temperature in biomolecular simulations. J. Chem. Theory Comput. 2010, 6, 2045-2058.

(13) Pastor, R. W.; Brooks, B. R.; Szabo, A. An analysis of the accuracy of Langevin and molecular dynamics algorithms. Mol. Phys. 1988, 65, 1409-1419.

(14) Hairer, E.; Wanner, G.; Lubich, C. Geom. Numer. Integr.; Springer-Verlag: Berlin/Heidelberg, 2006; pp 179-236.

(15) Sega, M.; Fábián, B.; Horvai, G.; Jedlovszky, P. How is the surface tension of various liquids distributed along the interface normal? J. Phys. Chem. C 27468-27477.

(16) Sega, M.; Fábián, B.; Jedlovszky, P. Pressure profile calculation with mesh Ewald methods. J. Chem. Theory Comput. 2016, 12, 4509-4515. 
(17) Schofield, P.; Henderson, J. Statistical mechanics of inhomogeneous fluids. Proc. Royal Soc. London A 1982, 379, 231-246.

(18) Pártay, L. B.; Hantal, G.; Jedlovszky, P.; Vincze, Á.; Horvai, G. A new method for determining the interfacial molecules and characterizing the surface roughness in computer simulations. Application to the liquid-vapor interface of water. J. Comput. Chem. 2008, 29, 945-956.

(19) Sega, M.; Fábián, B.; Jedlovszky, P. Layer-by-layer and intrinsic analysis of molecular and thermodynamic properties across soft interfaces. J. Chem. Phys. 2015, 143, 114709.

(20) Rowlinson, J. S.; Widom, B. Molecular theory of capillarity; Dover Publications Inc.: Mineola, New York, 1982.

(21) Gloor, G. J.; Jackson, G.; Blas, F. J.; de Miguel, E. Test-area simulation method for the direct determination of the interfacial tension of systems with continuous or discontinuous potentials. J. Chem. Phys. 2005, 123, 134703.

(22) Ghoufi, A.; Malfreyt, P. Calculation of the surface tension and pressure components from a non-exponential perturbation method of the thermodynamic route. J. Chem. Phys. 2012, 136, 024104.

(23) Hermans, J.; Berendsen, H. J. C.; van Gunsteren, W. F.; Postma, J. P. M. A consistent empirical potential for water-protein interactions. Biopolymers 1984, 23, 1513-1518.

(24) Jorgensen, W. L.; Chandrasekhar, J.; Madura, J. D.; Impey, R. W.; Klein, M. L. Comparison of simple potential functions for simulating liquid water. J. Chem. Phys. 1983, 79, 926 .

(25) Abascal, J. L.; Vega, C. A general purpose model for the condensed phases of water: TIP4P/2005. J. Chem. Phys. 2005, 123, 234505. 
(26) Mahoney, M. W.; Jorgensen, W. L. A five-site model for liquid water and the reproduction of the density anomaly by rigid, nonpolarizable potential functions. J. Chem. Phys. 2000, 112, 8910-8922.

(27) Rick, S. W. A reoptimization of the five-site water potential (TIP5P) for use with Ewald sums. J. Chem. Phys. 2004, 120, 6085.

(28) Abraham, M. J.; Murtola, T.; Schulz, R.; Páll, S.; Smith, J. C.; Hess, B.; Lindahl, E. GROMACS: High performance molecular simulations through multi-level parallelism from laptops to supercomputers. SoftwareX 2015, 1, 19-25.

(29) Plimpton, S. Fast parallel algorithms for short-range molecular dynamics. J. Comput. Phys. 1995, 117, 1-19.

(30) Limbach, H.-J.; Arnold, A.; Mann, B. A.; Holm, C. ESPResSo-an extensible simulation package for research on soft matter systems. Comput. Phys. Comm. 2006, 174, $704-727$.

(31) Ryckaert, J.-P.; Ciccotti, G.; Berendsen, H. J. Numerical integration of the cartesian equations of motion of a system with constraints: molecular dynamics of n-alkanes. J. Comput. Phys. 1977, 23, 327-341.

(32) Miyamoto, S.; Kollman, P. A. SETTLE: an analytical version of the SHAKE and RATTLE algorithm for rigid water models. J. Comput. Chem. 1992, 13, 952-962.

(33) Essmann, U.; Perera, L.; Berkowitz, M. L.; Darden, T.; Lee, H.; Pedersen, L. G. A smooth particle mesh Ewald method. J Chem Phys 1995, 103, 8577-8593.

(34) Hockney, R. W.; Eastwood, J. W. Computer simulation using particles; Taylor and Francis: New York, 1988; pp 267-304.

(35) Berendsen, H. J.; Postma, J. v.; van Gunsteren, W. F.; DiNola, A.; Haak, J. Molecular dynamics with coupling to an external bath. J. Chem. Phys. 1984, 81, 3684-3690. 
(36) Nosé, S. A molecular dynamics method for simulations in the canonical ensemble. Mol. Phys. 1984, 52, 255-268.

(37) Hoover, W. G. Canonical dynamics: Equilibrium phase-space distributions. Phys. Rev. A 1985, 31, 1695-1697.

(38) Sega, M.; Kantorovich, S. S.; Jedlovszky, P.; Jorge, M. The generalized identification of truly interfacial molecules (ITIM) algorithm for nonplanar interfaces. J. Chem. Phys. 2013, 138, 044110.

(39) Pártay, L. B.; Horvai, G.; Jedlovszky, P. Temperature and pressure dependence of the properties of the liquid-liquid interface. A computer simulation and identification of the truly interfacial molecules investigation of the water-benzene system. J. Phys. Chem. C 2010, 114, 21681-21693. 